\title{
外肢体机器人研究现状及发展趋势*
}

\author{
荆泓玮 朱延河 赵思恺 张清华 赵 杰 \\ (哈尔滨工业大学机器人技术与系统国家重点实验室 哈尔滨 150001)
}

\begin{abstract}
摘要: 外肢体机器人是一种新型的可穿戴人体辅助设备, 通过与人类肢体的对接融合、互助协作来提高人体活动、感知、操 作等能力, 在工业生产、医疗康复、助老助残及生活服务等领域都有着广阔的应用前景。分别针对当前国内外的外肢体机器 人研究情况, 对其本体结构、控制方法、应用领域等方面进行了综述。根据不同的本体结构与功能将外肢体机器人分为外肢 体与外手指; 根据结构柔顺特性将外肢体机器人分为刚性外肢体机器人与柔性外肢体机器人; 归纳分析了外肢体机器人的控 制方式，主要包括肢体映射控制、肌电信号控制与脑机接口控制; 分析总结了外肢体机器人面临的技术挑战，并对外肢体机 器人的技术发展趋势做出了展望。
\end{abstract}

关键词: 可穿戴机器人; 外肢体; 外手指; 人机交互; 人机协作; 人机共融

中图分类号: TP242

\section{Research Status and Development Trend of Supernumerary Robotic Limbs}

\section{JING Hongwei ZHU Yanhe ZHAO Sikai ZHANG Qinghua ZHAO Jie}

(State Key Laboratory of Robotics and System, Harbin Institute of Technology, Harbin 150001)

\begin{abstract}
Supernumerary robotic limbs are a new type of wearable human auxiliary equipment. Human's ability of activity, perception and operation can be improved through cooperation with supernumerary robotic limbs. The broad application prospect of supernumerary robotic limbs is shown in the fields of industrial production, medical rehabilitation, elderly and disabled assistance and life services. Aiming at the current research situation of supernumerary robotic limbs at home and abroad, the noumenon structures, control methods and application fields are reviewed. According to different noumenon structures and functions, supernumerary robotic limbs are divided into extra limbs and extra fingers. From the aspect of structural flexibility, supernumerary robotic limbs are divided into rigid supernumerary robotic limbs and flexible supernumerary robotic limbs. The current control methods of supernumerary robotic limbs are summarized and analyzed, including limb mapping control, EMG signal control and brain-machine interface control. The technical challenges and trends ahead of supernumerary robotic limbs are also discussed.
\end{abstract}

Key words: wearable robotics; extra limbs; extra fingers; human-robot interaction; human-robot cooperation; human-robot integration

\section{0 前言}

外肢体机器人是一种区别于假肢与外骨骼机器 人的新型可穿戴式机器人。不同于对人类肢体进行 增强的外骨骼机器人，外肢体机器人是对人类肢体 的拓展，通过机械肢体与人类肢体的对接融合、互 助协作来提升人体的活动、感知和操作等能力。

外肢体机器人的研究开始于 2012 年, 其中美国 麻省理工学院针对外肢体机器人开展了最为系统的

* 国家重点研发计划资助项目(2018YFB1305400)。20191118 收到初稿, 20200116 收到修改稿
理论研究, 并与波音公司合作开展了面向飞机制造装 配的应用研究 ${ }^{[1-5]}$, 综合水平较为领先。美国、日本 等地其他研究机构面向助老助残、医疗康复、日常辅 助及生活娱乐等方面开展了初步的研究 ${ }^{[6-25]}$ 。国内对 于外肢体机器人的研究起步较晚, 主要有哈尔滨工业 大学、华南理工大学、南开大学等研究机构, 初步地 提出了外肢体机器人本体结构设计概念 ${ }^{[18-20,25-26] 。}$

本文针对目前国内外具有代表性的外肢体机 器人, 分别从本体结构及应用、控制方法等方面 进行综述, 探讨分析目前外肢体机器人所面临的 主要技术挑战, 并对外肢体机器人技术发展趋势 做出展望。 


\section{1 外肢体本体结构及应用}

目前, 国内外的外肢体机器人按本体结构分类 主要分为外肢体和外手指, 应用于工业生产、助老 助残及生活服务等领域。

\section{1 外肢体}

外肢体主要通过模仿人体的四肢功能来拓展和 提高人体活动与操作能力。

美国麻省理工学院 ASADA 的研究团队国际上 最早开展外肢体的相关研究, 对于外肢体的研究系 统性较强, 有着较为领先的综合水平。为了能够解决 技术工人在飞机制造环境中, 长时间执行复杂精准安 装操作任务所带来的人体负荷问题, ASADA 的研究 团队提出了外肢体(Supernumerary robotic limb, SRL) 的设计想法, 并开展了一系列的研究工作 ${ }^{[1-5,27-31]}$ 。

该团队于 2012-2013 年设计了一款穿戴式 外肢体机器人(图 1a), 并提出了外肢体 (SRL)的概 念 ${ }^{[1-4]}$ 。此外肢体由两个独立的机械手臂组成, 穿戴 于人体腰部位置, 为穿戴者提供了第三臂和第四臂, 可以充当手臂或者腿来帮助穿戴者定位物体、举起 重物和保持平衡。此种外肢体设计的目的是增加穿 戴者执行操作时的力量和精度、扩展穿戴者操作范 围以及降低穿戴者工作负荷。

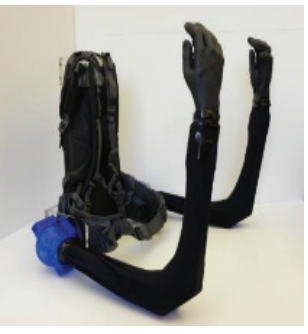

(a) 协作钻孔 $\mathrm{SRL}^{[1]}$

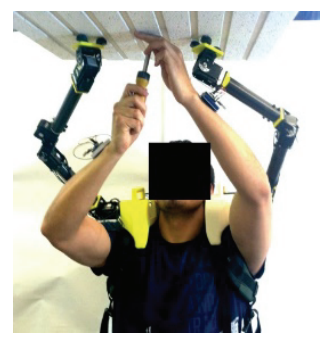

(c) 头顶工作 $\mathrm{SRL}^{[30]}$
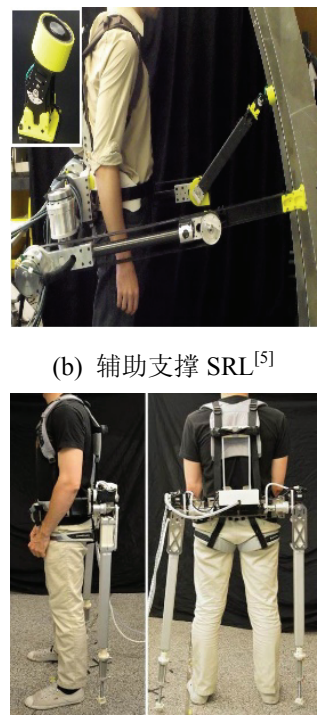

(d)下肢支撑 SRL ${ }^{[29]}$ (b) 辅助支撑 $\mathrm{SRL}^{[5]}$

图 1 麻省理工外肢体

PARIETTI 等于 2014 年将该腰部穿戴式外肢体 进一步改进(图 1b)。在慰部周围固定额外的两只手 臂，一只手臂用于人体和墙壁间支撑，另一只手臂 辅助人体进行钻孔工作 ${ }^{[5,31]}$ 。该设备通过支撑穿戴 者的身体和引导双手钻孔, 提高了钻孔作业的稳定
性和准确性, 被设计应用于常规工业机器人无法有 效执行的飞机装配任务中。

2014 年, ASADA 团队提出了一种穿戴于人体 肩部位置的外肢体机器人(图 1c), 本体结构为两只 刚性机械臂 ${ }^{300}$ 。此外肢体用于协助穿戴者在头顶工 作空间中执行任务, 能够主动与穿戴者进行紧密合 作，从而简化操作，减少穿戴者的工作量。

ASADA 团队于 2015-2016 年设计了腰部穿戴

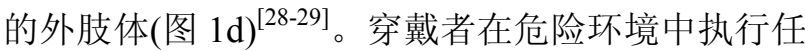
务或保持不适工作姿态时，此外肢体可以通过抵住 墙壁、抓住栏杆或针定地地面等方式来支撑人体。 此外肢体为制造业工人提供帮助，使其能够安全、 舒适、稳定地完成任务, 增强了安全性和舒适性。

日本庆应大学 SASAKI 等 ${ }^{[10-11]}$ 于 2017 年研制 了一款基于腿部和脚部动作信号控制的外肢体机器 人(图 2)。此外肢体机器人将人体腿部运动映射到外 肢体手臂，以实现外肢体与穿戴者协作来实现 “四 臂” 协同作业，从而拓展穿戴者日常活动能力并提 升了工作效率。

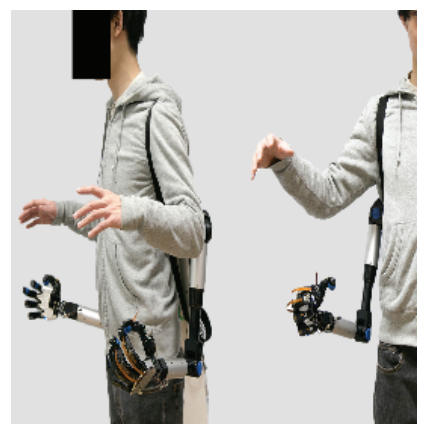

图 2 日本庆应大学外肢体 ${ }^{[11]}$

瑞典皇家理工学院 HAIDERK 等 ${ }^{[32]}$ 于 2015 年 提出了一种用于工业与生活的多用途外肢体机器人 (Multipurpose supernumerary robotic limb, MSRL)。 MSRL 是一种基于 SRL 概念的新型多用途外肢体机 器人, 其总体重量轻、便携性好。MSRL 可以重复 实现已记录的用户动作，配合其完成工作任务并进 行精度调整, 在工业生产领域与日常生活领域都有 着广泛的应用前景。

美国康奈尔大学 HOFFMAN 等 ${ }^{[33]} 2017$ 年设 计了一种穿戴于前臂部位的外肢体机器人(图 3), 本 体质量轻, 可实现伸缩动作, 并具有一定的抓取能 力。进行了外肢体抓取杯子的演示实验, 并对此种 外肢体的结构特点、穿戴者期望的控制方法和应用 场合进行了分析。

美国佐治利亚理工学院 WEINBERG 等于 2016 年设计了一种穿戴于肩部的外肢体架子鼓手臂 (图 4) ${ }^{[6-7,14]}$ 。该设备能够在用户操控的基础上, 通 
过接收音乐信息, 以不同的节奏和行动做出相应的 反应, 与穿戴者进行互动, 对于音乐家的演奏有所 帮助。

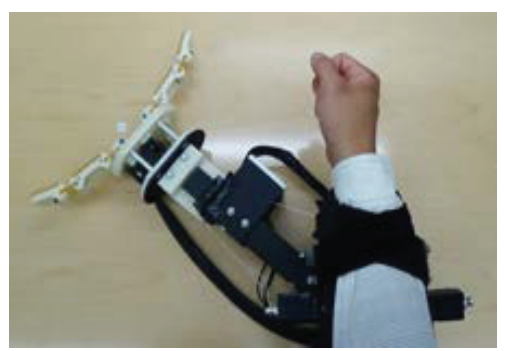

图 3 美国康奈尔大学外肢体 ${ }^{[33]}$

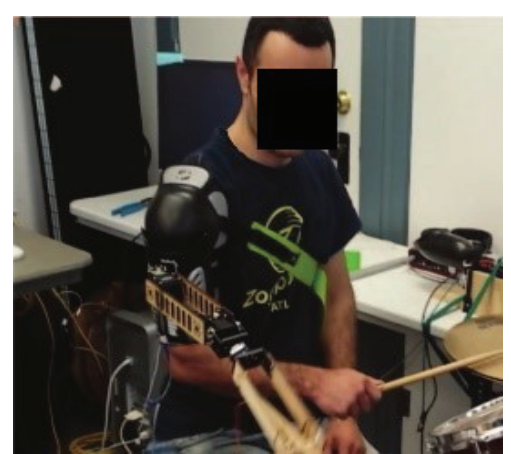

图 4 佐治利亚理工学院外肢体架子鼓手臂 ${ }^{[14]}$

随着对于外肢体灵巧化、轻量化水平等要求的 提高, 在刚性外肢体机器人基础之上, 各国研究机 构对柔性的外肢体机器人进行了研究。

美国亚利桑那州立大学 NGUYEN 等 ${ }^{[34]}$ 于 2018 年提出了一种面向日常生活辅助的柔性外肢体(Soft Poly-Limbs, SPL)(图 5a), 结构上与象鼻类似, 能 够举起 2.35 倍于自身重量的物体。SPL 有着高灵活 度和高柔顺性的特点, 具有与穿戴者安全交互, 以 及在工作和日常生活中协助完成各种任务的能力。

AL-SADA 等 ${ }^{[35]}$ 于 2019 年提出了一种在日常生 活中使用的多功能蛇形外肢体 Orochi(图 5b)。Orochi 仿蛇形的形态进行设计, 有着质量轻、灵活性高的 特点。以不同的工作形式展现了 Orochi 的工作能力, 并对其在日常生活中的多用途使用场景、基于周围 环境的可穿戴性和生活中的应用性进行了分析。

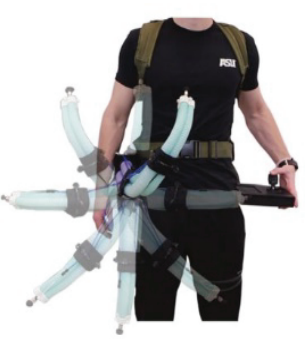

(a) $\mathrm{SPL}^{[34]}$

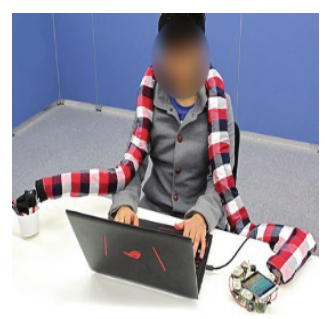

(b) Orochi ${ }^{[35]}$

图 5 柔性外肢体

\section{2 外手指}

外手指是一类仿手指的外肢体机器人, 能够与 人体手部或臂部等部位协同工作实现夹持物体、 提高操作能力等功能。目前外手指包括刚性外手 指与柔性外手指, 主要应用于助老助残、生活服务 等领域。

美国麻省理工学院 WU 与 ASADA 于 2014 年 设计了一种刚性的外手指 SR Fingers(图 6a) ${ }^{[36-37]}$, 用 以辅助帮助截肢和中风患者在只有单手操作能力的 情况下维持独立的生活。SR Fingers 由穿戴于手腕 部位的两根机械手指组成, 能够辅助穿戴者实现拧 下瓶盖、打开容器盖子等操作任务。验证了外手指 具有提高手部缺陷患者独立生活能力和工作效率的 可能性。

麻省理工学院媒体实验室 LEIGH 等于 2016 年 设计了一种穿戴于手腕的外手指 Extra Fingers (图

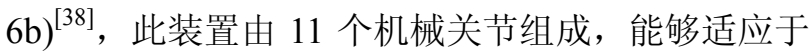
不同任务的应用。在文中进行了此外手指在摄影、 提水桶、提供书写平台等日常生活辅助的多用途功 能演示。

意大利技术研究院 HUSSAIN 等于 2014 年提出 了一种四自由度模块化的第六根手指 Sixth-

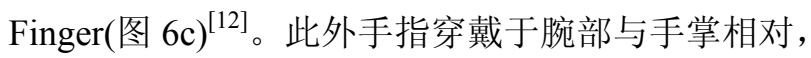
通过分析人体抓取过程中的手部运动配合手部动 作, 扩大手的工作空间, 增强穿戴者的抓取能力。

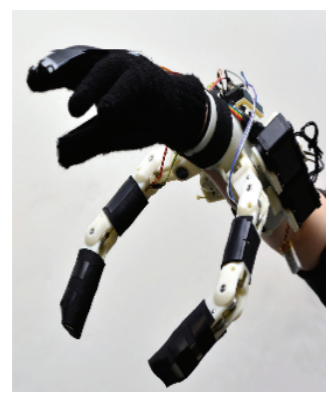

(a) 助残外手指 ${ }^{[36]}$

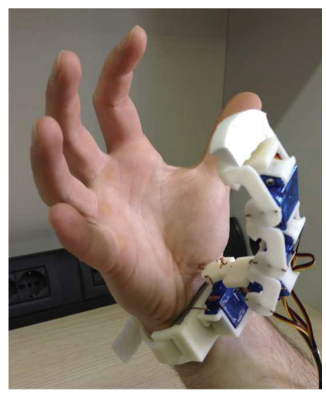

(c) 第六根手指 ${ }^{[12]}$

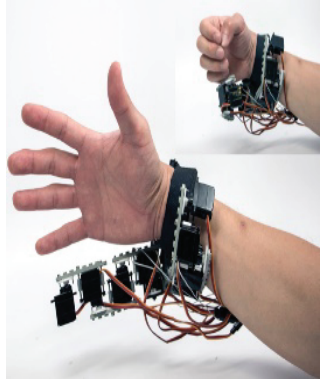

(b) 生活辅助外手指 ${ }^{[38]}$

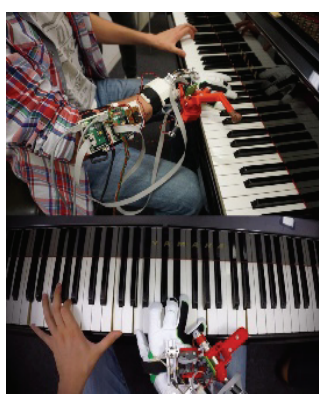

(d) 钢琴演奏外手指 ${ }^{[39]}$
图 6 刚性外手指

英国伦敦帝国学院 CUNNINGHAM 等 ${ }^{[39]}$ 于 2018 年设计了一种辅助钢琴演奏的外手指 SR3T 
(图 6d)。本体结构是装有一根机械手指的手套, 通 过与穿戴者其他手指协作, 扩展穿戴者所能碰触的 音阶范围, 增强音乐家弹奏钢琴的能力。主要以此 种应用于音乐领域的外手指来验证穿戴者有着适应 外肢体的可能性。

柔性外手指是在国际上各研究机构的学者研制 了刚性结构的外手指后, 提出的更加灵活、轻便以 及抓取适应性强的柔性外手指机器人。

麻省理工学院 TIZIANI 等 ${ }^{[40]}$ 于 2017 年设计了 一种气压驱动的模块化变刚度外手指(图 7a), 并进行 了其辅助抓取能力的试验测试。此种柔性外手指由软 体手指指骨和变刚度气动弯曲执行器组成, 有着较好 的欠驱动抓取适应能力以及分析经验数据预测抓取 能力, 为柔性外手指的后续研究提供理论基础。

麻省理工学院媒体实验室 HU 等 ${ }^{[41]}$ 于 2017 年设 计了一种由线绳和伺服电动机驱动的柔性外手指 (图 7b)。目的是为了解决刚性外手指的重量问题和 对其他部位的干扰问题, 提高装置的柔顺性。文中 演示了应用此外手指对智能手机上的对象进行触摸 交互、键盘输入等操作。

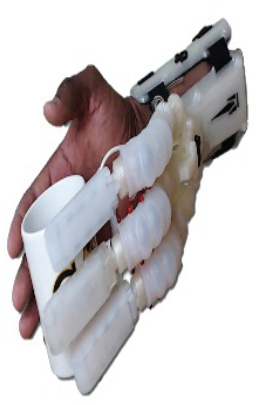

(a) 气动外手指 ${ }^{[40]}$

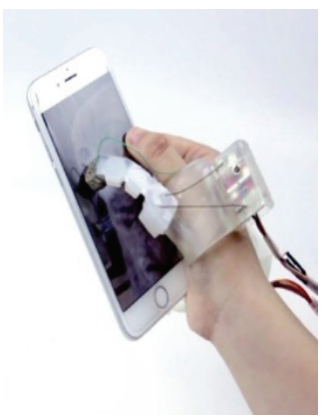

(b) 线驱动外手指 ${ }^{[41]}$

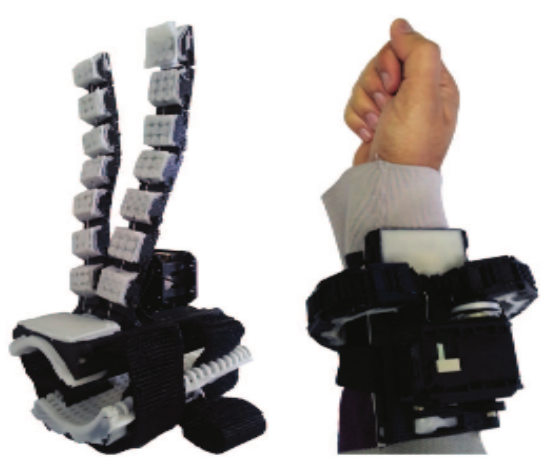

(c) 柔性第六指 ${ }^{[13]}$

图 7 柔性外手指

意大利技术研究院 HUSSAIN 等 ${ }^{[9,13]}$ 于 2017 年 在第六根手指原型的基础上提出了柔性第六指 Soft-Sixth Finger(图 7c)。柔性第六指主要由一个由 线绳驱动的柔性手指和一个支撑基组成, 每个模块 由 $3 \mathrm{D}$ 打印的柔性材料构成, 有着高弹性的反复运
动特性和减振效果。该装置主要为了帮助上肢偏瘫 患者实现抓取功能, 在慢性脑卒中患者穿戴试验中 成功地辅助患者完成水杯抓取、罐头开启等各种任 务, 提高了患者自主操作能力。

\section{3 外肢体国内相关研究}

哈尔滨工业大学朱延河等 ${ }^{[18]}$ 于 2018 年提出一 种穿戴式变构型外肢体机器人, 其穿戴位置为背部。 提出了一种串并联变构型的本体结构方案, 根据不 同作业需求具有两种工作模式, 分为串混联型工作 方式和并联型工作方式。串联结构有着工作空间大、 运动灵活特点, 有着辅助支撑、抓持等功能; 并联 结构的大出力、高刚度特性, 实现负载、精细作业。 此外肢体在军事演练、消防营救等领域有着良好的 应用前景。此后提出了一种基于气压驱动连续体的 可穿戴柔性辅助操作臂 ${ }^{[19]}$, 本体结构轻便灵活, 能 够适应不同环境, 在灾后救援、医疗康复及人体复 杂操作辅助等方面有着应用前景。

南开大学段峰等 ${ }^{[20]}$ 于 2018 年提出了一种外肢 体辅助作业机械臂, 以类似人类外肢体结构进行设 计, 未端能够夹紧不规则物体, 在辅助作业领域有 着应用前景。

华南理工大学杨辰光等 ${ }^{[25]}$ 于 2015 年提出一种 肩部可穿戴功能辅助机械臂, 为穿戴者在工作中提 供额外协助, 降低劳动强度, 提高工作效率, 在残 障人士的生活辅助方面有着应用前景。

\section{2 外肢体控制方法}

外肢体机器人只有采用可靠高效的控制方法, 才能更好地实现人机协作与人机共融, 提升穿戴者 的感知、操作等能力。在外肢体的控制方法上, 美 国麻省理工学院的 LEIGH 等 ${ }^{[42]}$ 结合当时国际上部 分外肢体机器人对于人机共融问题进行了探讨分 析, 将机器人增强设备的控制方法按照自主程度分 为: 直接控制、伪影射控制、辅助控制、共享控制。 此种分类方法较为概括, 本文将目前外肢体控制方 法具体地按照控制复杂程度分为肢体映射控制、肌 电信号控制和脑机接口控制。

\section{1 肢体映射控制}

肢体映射控制是指将对外肢体机器人的控制与 穿戴者肢体动作或肢体位置相关联, 建立映射关系 的一类控制方法。

麻省理工学院 BONILLA 与 ASADA 针对其设 计的肩部穿戴外肢体机器人, 提出了一种基于演示 与学习的控制算法 ${ }^{[30]}$ 。演示时, 操作者穿戴外肢体 机器人在他人辅助的情况下完成所需要执行的任 
务, 采集操作者与外肢体机器人的运动信号。采用 CPN 和 PLS 混合的方式进行学习并建立映射关系, 使其能够根据操作者肢体运动信号迅速做出下一步 动作的预判处理。

WU 等 ${ }^{[36]}$ 基于一种外手指机器人探讨了两种控 制方式, 一个能够在不同运动模式之间进行离散的 转换, 另一个则连续地控制外手指进行抓取行为。 此种控制方法是通过穿戴者肘部惯性传感器的运动 信号来与机器手指建立映射关系, 实现 “保持和操 纵” 任务。WU 等 ${ }^{[16]}$ 后续研究中考虑了外手指机器 人在应用过程中的人为干扰问题, 提出了一种数据 驱动的潜空间阻抗控制方法, 使机器人在操作过程 中既能保护操作物体, 又能实现人体的自然运动。 仿真和试验证明了潜空间阻抗控制器在外手指辅助 下实现单手物体操纵的有效性。

瑞典皇家理工学院 HAIDERK 等 ${ }^{[32]}$ 提出了两种 应用于多用途外肢体机器人 MSRL 的控制方法: 主从 跟随方法和图形用户界面方法。主从跟随方法是由控 制器记录人体手臂运动位置, 建立与机器手臂映射关 系, 反复执行相同任务。第二种方法指依靠编程实现 机器人精准角度或位移运动时的控制方式。

日本庆应大学的 SASAKI 等 ${ }^{[11]}$ 基于所研制的外 肢体机器人, 提出了一种具有高自由度肢体运动与 控制的多臂交互系统 MetaLimbs。穿戴者的脚部位 置动作映射于机器人的手臂位置运动, 脚趾与机器 人手部位置建立映射。建立了完整的肢体映射控制 方法, 实现外肢体高自由度的运动控制。

伦敦帝国学院 CUNNINGHAM 等 ${ }^{[39]}$ 根据其设 计的用于钢琴演奏外手指, 提出了左脚背屈动作映 射外肢体垂直运动的控制方法。经过试验, 能够实 现可穿戴钢琴手指的有效控制, 有着较好的适应性。

\section{2 肌电信号控制}

人体肌电信号是人体肌肉在收缩时所伴随的电信 号, 是目前无创检测肌肉活动的一种重要方法。目前国 际上研究人员主要应用不干涉穿戴者执行主要操作活 动肌肉的肌电信号来控制外肢体机器人的运动。

麻省理工学院 ASADA 等 ${ }^{[27]}$ 提出了一种应用人 体躯干胸肌和腹肌的肌电信号控制外肢体的方法。 选择收集胸部和腹部的肌电信号是因为相较于采集 身体的其他部位的信号, 采集胸部腹部的肌电信号 更不容易干扰实际操作, 也不容易影响到穿戴者的 日常生活。但此种胸肌和腹肌的肌电信号控制方法 只能控制 2 自由度的外肢体，有着局限性。

麻省理工学院媒体实验室 LEIGH 等 ${ }^{[38]}$ 提出了 一种应用人体肱桡肌(肘关节附近的前臂肌肉)的肌
电信号控制外手指的方法。经过 13 种常用手势的肌 电信号数据分析, 发现肱桡肌与这些手势相关性很 低, 可以作为一种不干涉正常手部运动的外肢体控 制方式。在随后试验中, 实现了用该部分肌肉的肌 电信号进行外肢体控制的抓取任务。

意大利技术研究院 HUSSAIN 等 ${ }^{[8]}$ 设计了一种智 能帽子(eCap)来收集穿戴者额肌的肌电信号，从而控 制外手指。此外手指设计对象是中风患者, 他们的额 肌收缩控制是正常的，因此，该控制方法相对有效。

美国佐治利亚理工学院 WEINBERG 等 ${ }^{[7]}$ 基于 一种外肢体架子鼓手臂, 设计了由尺侧腕伸肌和桡 侧腕屈肌的肌电信号实现敲击的控制方法, 并加入 了学习算法, 能够根据敲击节奏来增加额外的音符。

日本名古屋大学 HASEGAWA 等 ${ }^{[21-23]}$ 提出了用 耳后肌的肌电信号结合电刺激触觉感知反馈来控制 外手指。对比了耳后肌、尺侧腕伸肌和鱼际肌的肌 电信号控制外手指的效果，验证了耳后肌的控制效 果更好的猜想。在后续的研究中 ${ }^{[15,17]}$, 用拇指尖电 刺激触觉反馈控制的试验, 验证了感知反馈在外肢 体机器人控制上的重要性。

\section{3 脑机接口控制}

脑机接口(Brain-machine interface, BMI)控制是 一种利用人体脑电波信号实现机器控制的方法, 一 直受到国内外学者广泛关注。CARMENA 等 ${ }^{[43]}$ 于 2003 年首次证明了入侵式 BMI 应用于非人类灵长 类动物的可行性; HOCHBERG 等 ${ }^{[44]}$ 于 2012 年首次 将其应用于四肢㿈疾的人，证明人体脑机接口控制 的可行性。将 BMI 控制应用于外肢体机器人上是最 为直接的, 但复杂程度和难度都很高。如何更好地 实现外肢体机器人的 BMI 控制进而实现人机协同 控制也是国际上学者的主要研究内容。

CHRISTIAN 等 ${ }^{[45]}$ 于 2018 年提出了一种用非入 侵式 BMI 控制第三只手臂的方法, 并进行了演示及 试验效果分析。试验结果表明影响 BMI 控制外肢体 手臂的主要因素有机器人的视觉外观、参与者的协 调能力和注意力水平、感知反馈问题等。文中揭示 了人类大脑不仅能够控制外部设备, 而且能够在多 任务的情况下进行注意力分配的调节。

目前而言, 关于 BMI 控制的研究还在进行中, 并存在着增加人脑的操作负荷、易发生误判操作情 况、操作延时响应高等缺陷 ${ }^{[46]}$ 。如何将其更好地应 用于外肢体机器人还有待于研究与完善。

目前国际上具有代表性的外肢体如表 1 所示, 主要从外肢体结构、穿戴部位、控制方法和辅助类 型方面对所述外肢体机器人进行归纳。 
表 1 外肢体机器人分类总结

\begin{tabular}{|c|c|c|c|c|}
\hline 研究机构 & 外肢体结构 & 穿戴部位 & 控制方法 & 辅助类型 \\
\hline \multirow{7}{*}{ 麻省理工学院 } & 刚性外肢体 ${ }^{[1-5,31]}$ & 腰部 & & 作业辅助 \\
\hline & 刚性外肢体 ${ }^{[30]}$ & 肩部 & 肢体映射控制 & 作业辅助 \\
\hline & 刚性外肢体 ${ }^{[28-29]}$ & 腰部 & & 作业辅助 \\
\hline & 刚性外手指 ${ }^{[31,36]}$ & 前臂 & 肢体映射控制 & 生活辅助 \\
\hline & 刚性外手指[38] & 前臂 & 肌电信号控制 & 生活辅助 \\
\hline & 柔性外手指[40] & 前臂 & & 生活辅助 \\
\hline & 柔性外手指 ${ }^{[41]}$ & 前臂 & & 生活辅助 \\
\hline 庆应大学 & 刚性外肢体 ${ }^{[1]}$ & 腰部 & 肢体映射控制 & 生活辅助 \\
\hline 瑞典皇家理工 & 刚性外肢体 ${ }^{[32]}$ & 腰部 & 肢体映射控制 & 作业辅助、生活辅助 \\
\hline 康奈尔大学 & 刚性外肢体 ${ }^{[33]}$ & 前臂 & & 作业辅助、生活辅助 \\
\hline 意大利技术研究院 & 柔性外手指[8-9, 13] & 前臂 & 肌电信号控制 & 生活辅助 \\
\hline 佐治利亚理工学院 & 刚性外肢体 ${ }^{[7]}$ & 肩部 & 肌电信号控制 & 生活辅助 \\
\hline 伦敦帝国学院 & 刚性外手指[39] & 手部 & 肢体映射控制 & 生活辅助 \\
\hline 亚利桑那州立大学 & 柔性外肢体 ${ }^{[34]}$ & 背部 & & 作业辅助、生活辅助 \\
\hline 早稻田大学 & 柔性外肢体 ${ }^{[35]}$ & & & 生活辅助 \\
\hline \multirow{2}{*}{ 哈尔滨工业大学 } & 刚性外肢体 ${ }^{[18]}$ & 背部 & & 作业辅助 \\
\hline & 柔性外肢体 ${ }^{[19]}$ & 背部 & & 作业辅助、生活辅助 \\
\hline 南开大学 & 刚性外肢体 ${ }^{[20]}$ & & & 作业辅助 \\
\hline 华南理工大学 & 刚性外肢体 ${ }^{[25]}$ & 背部 & & 作业辅助 \\
\hline
\end{tabular}

\section{3 技术挑战及发展趋势}

外肢体机器人的研究目前处于起步阶段, 仍有 诸多问题未被解决, 需要进一步的探讨与研究。通 过对外肢体技术研究现状的梳理与分析, 总结出外 肢体研究面临的技术挑战及发展趋势主要体现在以 下三个方面。

（1）外肢体的结构设计方面。外肢体的实用性 和人体穿戴舒适度是研究人员需要思考的主要问 题。外肢体机器人由最初的刚性结构发展到柔性结 构, 穿戴部位则由腰部、肩部发展到手臂等部位。 未来外肢体机器人将逐步向刚柔一体化结构的方向 发展; 需要对外肢体进行合理穿戴接口设计, 并提 高外肢体负载自重比; 提高穿戴的舒适性; 使外肢 体有多种形态的变化; 拥有更多末端工具接口, 实 现功能多样化。

(2) 外肢体的交互控制技术方面。外肢体的交 互控制技术是当前的技术挑战之一。为了实现人机 交互, 已经提出了肢体映射控制、肌电信号控制和 脑机接口控制等控制方法。采用肢体映射控制等方 法虽然更加简单可靠, 但可能会影响人类肢体的正 常活动。脑机接口控制方法最为直接, 但十分复杂, 存在着脑负荷高、响应时间长、易发生误判等问题。 增加外肢体机器人的感知反馈, 将实现更有效的控
制。此外, 外肢体机器人的控制方式应当不同于非 穿戴机械臂, 可以模仿人类操作模式, 在视觉等信 息的引导下, 形成肢体与对象之间的反馈控制系统。 因此, 充分利用人体有限自由度, 结合多重感知反 馈机制及多种控制方法, 实现人机之间信息的双向 传递将是未来的发展趋势。

（3）外肢体的人机协作技术方面。外肢体与人 进行协同工作的过程中, 要避免与人体发生干涉碰 撞。因此, 建立人与外肢体系统协同工作机制、形 成可靠的执行策略方法、保证人身安全与设备安全、 发挥出外肢体机器人最大的人机协作效能是目前需 要面对的挑战。随着人工智能的兴起与发展, 外肢 体机器人也将不仅仅是执行单纯的控制指令, 而是 有着学习能力、能够掌握多种技能、适应不同的任 务场景, 有着更多更广的应用领域。

\section{4 总结与展望}

目前外肢体机器人按照本体结构分为外肢体、 外手指。外肢体的设计应用领域由最初的工业生产 领域逐步发展向生活服务等多用途领域。外手指相 对于外肢体有着小巧轻便的特点, 主要设计应用于 医疗康复、生活服务等领域。柔性外肢体相对于刚 性外肢体, 具有重量轻、灵活性高和柔顺性好等优 点, 所能应用的领域也更加广阔多样。外肢体机器 
人的结构设计逐渐向着刚柔一体化、功能多样化的 方向发展。外肢体机器人的控制方法主要包括肢体 映射控制、肌电信号控制和脑机接口控制。肢体映 射是对与任务不相关肢体的利用; 肌电信号控制是 对人体冗余活动肌肉的利用; 脑机接口是最为直接 的人体意念控制。解决外肢体机器人的人机交互问 题, 真正实现人机协作用途至关重要。

外肢体机器人在国防军事、工业生产、医疗康 复、生活服务等诸多领域都有着广阔的应用前景。 其发展将为人机协作与共融的实现提供参考和技术 支持, 将在提升人民生活水平、加强社会安全保障、 改善社会公共服务等方面起到推动作用。外肢体机 器人的研究才刚刚起步, 其未知领域充满着可能性 与挑战, 需要不断地探索与完善。

\section{参 考 文 献}

[1] LLORENS-BONILLA B, PARIETTI F, ASADA H H. Demonstration-based control of supernumerary robotic limbs[C]// Intelligent Robots and Systems (IROS), 2012 IEEE/RSJ International Conference on. IEEE, 2012.

[2] DAVENPORT C, PARIETTI F, ASADA H H. Design and biomechanical analysis of supernumerary robotic limbs[C]// ASME Dynamic Systems \& Control Conference Joint with the JSME Motion \& Vibration Conference, 2012.

[3] PARIETTI F, ASADA H H. Dynamic analysis and state estimation for wearable robotic limbs subject to humaninduced disturbances[C]// Robotics and Automation (ICRA), 2013 IEEE International Conference on. IEEE, 2013.

[4] DAVENPORT C. Supernumerary robotic limbs: Biomechanical analysis and human-robot coordination training[J]. Massachusetts Institute of Technology, 2013(9): 787-793.

[5] PARIETTI F, ASADA H H . Supernumerary robotic limbs for aircraft fuselage assembly: Body stabilization and guidance by bracing[C]// 2014 IEEE International Conference on Robotics and Automation (ICRA). IEEE, 2014.

[6] GOPINATH D, WEINBERG G. A generative physical model approach for enhancing the stroke palette for robotic drummers[J]. Robotics and Autonomous Systems, 2016, 86: 207-215.

[7] BRETAN M, GOPINATH D, MULLins P, et al. A robotic prosthesis for an amputee drummer[J]. arXiv preprint arXiv: 1612.04391, 2016.

[8] HUSSAIN I, SALVIETTI G, SPAGNOLETTI G, et al.
A soft supernumerary robotic finger and mobile arm support for grasping compensation and hemiparetic upper limb rehabilitation[J]. Robotics and Autonomous Systems, 2017, 93: 1-12.

[9] SALVIETTI G, HUSSAIN I, CIONCOLONI D, et al. Compensating hand function in chronic stroke patients through the robotic sixth finger[J]. IEEE Transactions on Neural Systems and Rehabilitation Engineering, 2017, 25(2): $142-150$.

[10] SASAKI T, SARAIJI M Y, FERNANDO C L, et al. MetaLimbs: Metamorphosis for multiple arms interaction using artificial limbs[C]// ACM SIGGRAPH 2017 Posters. ACM, 2017.

[11] SASAKI T, SARAIJI M Y, FERNANDO C L, et al. MetaLimbs: Multiple arms interaction metamorphism[C] // ACM SIGGRAPH 2017 Emerging Technologies. ACM, 2017.

[12] PRATTICHIZZO D, MALVEZZI M, HUSSAIN I, et al. The sixth-finger: A modular extra-finger to enhance human hand capabilities[C]// Ro-man: the IEEE International Symposium on Robot \& Human Interactive Communication. IEEE, 2014.

[13] HUSSAIN i, SPAGNOLETTI G, SAlVietTi G, et al. Toward wearable supernumerary robotic fingers to compensate missing grasping abilities in hemiparetic upper $\operatorname{limb}[\mathrm{J}]$. The International Journal of Robotics Research, 2017, 36(13-14): 1414-1436.

[14] KHODAMBASHI R, WEINBERG G, SINGHOSE W, et al. User oriented assessment of vibration suppression by command shaping in a supernumerary wearable robotic arm[C]// 2016 IEEE-RAS 16th International Conference on Humanoid Robots (Humanoids). IEEE, 2016.

[15] ZHU Y, ITO T, AOYAMA T, et al. Development of sense of self-location based on somatosensory feedback from finger tips for extra robotic thumb control[J]. ROBOMECH Journal，2019，6(1): 7.

[16] WU F Y, ASADA H H. Decoupled motion control of wearable robot for rejecting human induced disturbances[C] // 2018 IEEE International Conference on Robotics and Automation (ICRA). IEEE, 2018.

[17] SEGURA MERAZ N, SOBAJIMA M, AOYAMA T, et al. Modification of body schema by use of extra robotic thumb[J]. ROBOMECH Journal，2018，5(1): 3.

[18] 朱延河, 张睿, 李相龙, 等. 穿戴式变构型外肢体机 器人: 中国, CN109015605A[P]. 2018-12-18. 
ZHU Yanhe, ZHANG Rui, LI Xianglong, et al. Wearable allosteric robotic limbs: China, CN109015605A[P]. 2018-12-18.

[19] 朱延河, 隋东宝, 王天铩, 等. 可穿戴柔性辅助操作 臂: 中国, CN108673471A[P]. 2018-10-19.

ZHU Yanhe, SUI Dongbao, WANG Tianshui, et al. Wearable flexible auxiliary operation limbs: China, CN108673471A[P]. 2018-10-19.

[20] 段峰, 刘振强, 史亮, 等. 一种外肢体辅助作业机械 臂: 中国, CN108453706A[P]. 2018-08-28.

DUAN Feng, LIU Zhenqiang, SHI Liang, et al. A type of robotic arms for auxiliary operation: China, CN108453706A[P]. 2018-08-28

[21] SHIKIDA H, NOEL S M, HASEGAWA Y. Acquisition of new body representation about extra robotic thumb by use of vestigial muscles[C]// 2017 IEEE International Conference on Cyborg and Bionic Systems (CBS). IEEE, 2017.

[22] SEGURA M N, SHIKIDA H, HASEGAWA Y. Auricularis muscles based control interface for robotic extra thumb $[\mathrm{C}] / / 2017$ International Symposium on Micro- NanoMechatronics and Human Science (MHS). IEEE, 2017: 1-3.

[23] SOBAJIMA M, SATO Y, XUFENG $W$, et al. Improvement of operability of extra robotic thumb using tactile feedback by electrical stimulation[C]// 2015 International Symposium on Micro-NanoMechatronics and Human Science (MHS). IEEE， 2015.

[24] HUSSAIN I, MELI L, PACCHIEROTTI C, et al. Vibrotactile haptic feedback for intuitive control of robotic extra fingers[C]// World Haptics Conference. IEEE, 2015.

[25] 杨辰光, 梁培栋, 陈君申, 等. 肩部可穿戴功能辅助 机械臂：中国，CN104825258A [P]. 2015-08-12.

YANG Chengguang, LIANG Peidong, CHEN Junshen, et al. Shoulder wearable auxiliary robotic limbs: China, CN104825258A [P]. 2015-08-12.

[26] WU Y, WU Z, FU C. Continuous arm gesture recognition based on natural features and logistic regression[J]. IEEE Sensors Journal, 2018, 18(19): 8143-8153.

[27] PARIETTI F, ASADA H H. Independent, voluntary control of extra robotic limbs[C]// 2017 IEEE International Conference on Robotics and Automation (ICRA). IEEE, 2017.

[28] PARIETTI F, ASADA H. Supernumerary robotic limbs for human body support[J]. IEEE Transactions on
Robotics, 2016, 32(2): 301-311.

[29] PARIETTI F, CHAN K C, HUNTER B, et al. Design and control of supernumerary robotic limbs for balance augmentation[C]// Proceedings IEEE International Conference on Robotics and Automation, 2015: 5010-5017.

[30] BONILLA B L, ASADA H H . A robot on the shoulder: Coordinated human-wearable robot control using coloured petri nets and partial least squares predictions[C] // 2014 IEEE International Conference on Robotics and Automation (ICRA). IEEE, 2014.

[31] PARIETTI F, CHAN K, ASADA H H. Bracing the human body with supernumerary robotic limbs for physical assistance and load reduction[C]// 2014 IEEE International Conference on Robotics and Automation (ICRA). IEEE, 2014.

[32] SRINIVAS S, VIRK G S, HAIDER U. Multipurpose supernumerary robotic limbs for industrial and domestic applications[C]// 2015 20th International Conference on Methods and Models in Automation and Robotics (MMAR). IEEE, 2015.

[33] VATSAL V, HOFFMAN G. Wearing your arm on your sleeve: Studying usage contexts for a wearable robotic forearm[C]// 2017 26th IEEE International Symposium on Robot and Human Interactive Communication (RO-MAN). IEEE, 2017.

[34] NGUYEN P H, SPARKS C, NUTHI S G, et al. Soft poly-limbs: Toward a new paradigm of mobile manipulation for daily living tasks[J]. Soft Robotics. 2018， 6(1): 38-53.

[35] AL-SADA M. Orochi: Investigating requirements and expectations for multipurpose daily used supernumerary robotic limbs[C]// Proceedings of the 10th Augmented Human International Conference 2019. ACM， 2019.

[36] WU F Y, ASADA H H. 'Hold-and-manipulate' with a single hand being assisted by wearable extra fingers[C] // Proceedings IEEE International Conference on Robotics and Automation, 2015: 6205-6212.

[37] WU F Y, ASADA H. Bio-artificial synergies for grasp posture control of supernumerary robotic fingers[M]. Cambridge: MIT Press, 2014.

[38] LEIGH S W, MAES P. Body integrated programmable joints interface[C]// $2016 \mathrm{CHI}$ Conference on Human Factors in Computing Systems. ACM, 2016.

[39] CUNNINGHAM J. The supernumerary robotic 3rd thumb for skilled music tasks[C]// 2018 7th IEEE International 
Conference on Biomedical Robotics and Biomechatronics (Biorob). IEEE, 2018.

[40] TIZIANI L, HART A, CAHOON T, et al. Empirical characterization of modular variable stiffness inflatable structures for supernumerary grasp-assist devices[J]. The International Journal of Robotics Research, 2017 , 36(13-14): 1391-1413.

[41] HU Y, LEIGH S, MAES P. Hand development kit: Soft robotic fingers as prosthetic augmentation of the hand[C] // 2017 30th Annual ACM Symposium on User Interface Software and Technology. ACM, 2017.

[42] LEIGH S, AGRAWAL H, MAES P. Robotic symbionts: Interweaving human and machine actions[J]. IEEE Pervasive Computing, 2018, 17(2): 34-43.

[43] CARMENA J M, LEBEDEV M A, CRIST R E, et al. Learning to control a brain-machine interface for reaching and grasping by primates[J]. PLOS Biology, 2003, 1(2): e42.

[44] HOCHBERG L R, DANIEL B, BEATA J, et al. Reach and grasp by people with tetraplegia using a neurally controlled robotic arm[J]. Nature, 2013, 485(7398): 372-375

[45] PENALOZA C I, NISHIO S. BMI control of a third arm for multitasking[J]. Science Robotics, 2018, 3(20): t1228.

[46] HE Y, EGUREN D, AZORÍN J M, et al. Brain-machine interfaces for controlling lower-limb powered robotic systems[J]. Journal of Neural Engineering，2018，15(2): 21004.

作者简介: 荆泓玮, 男, 1997 年出生, 博士研究生。主要研究方向为可 穿戴机器人。

E-mail: jhw_hit@163.com

朱延河(通信作者), 男, 1975 年出生, 博士, 教授, 博士研究生导师。 主要研究方向为外骨骼机器人, 外肢体机器人, 模块化自重构机器人。

E-mail: yhzhu@hit.edu.cn 